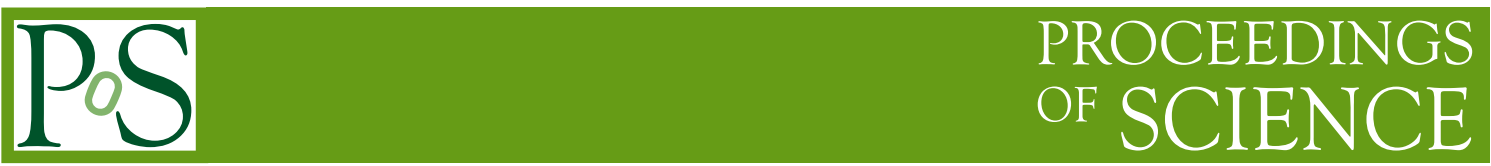

\title{
Highlights from ANTARES
}

\author{
Dorothea Samtleben* on behalf of the ANTARES collaboration \\ Nikhef, Amsterdam \& Leiden University \\ E-mail: dosamt@nikhef.nI
}

The underwater neutrino telescope ANTARES has been continuously operating since 2007 in the Mediterranean Sea. The transparency of the water allows for a very good angular resolution in the reconstruction of signatures of all flavours neutrino interactions. This results in unprecedented sensitivity for the search of astrophysical neutrino sources at TeV energies, in the Southern Sky, so that valuable constraints could be set on the origin of the cosmic neutrino flux discovered by the IceCube detector. Aside from a search for neutrino sources on the full sky dedicated searches have been performed for promising neutrino source candidates - among those the high energy neutrino candidates from IceCube - and several interesting regions like the Galactic Plane or the Fermi Bubble. ANTARES is also embedded in a manifold multi-messenger program with e.g. optical and X-ray follow-up observations of promising astrophysical neutrino candidates. External observations are also used by ANTARES in special analyses like a follow-up on Gamma Ray Burst events or the evaluation of possible neutrino signals correlated with the newly discovered gravitational wave signals. So far no significant correlation with external observations has been detected. Strong constraints could also be set on the dark matter cross section from the search of neutrinos from potential dark matter annihilation in massive objects like the Sun and the Galactic Centre.

XVII International Workshop on Neutrino Telescopes

13-17 March 2017

Venezia, Italy

${ }^{*}$ Speaker. 


\section{Introduction}

The high energy non-thermal Universe has been investigated over many energy decades via various messengers (photons, cosmic rays). Many open questions remain: since charged cosmic rays are deflected by magnetic fields their astrophysical sources have still not been identified. Many astrophysical sources have been seen by now in $\mathrm{GeV}$ to $\mathrm{TeV} \gamma$ rays observations, but the processes leading to their high energy emission are not yet understood and both leptonic as well as hadronic scenarios are equally viable.

Neutrinos have already several decades ago been indicated as valuable astrophysical messengers. Being neutral and only weakly interacting they can transverse dense media and travel undeflected long distances, thus keeping the information on their origin. A discovery of neutrino fluxes from astrophysical sources can unambigously confirm the hadronic origin of $\gamma$ ray emission and with this provide invaluable information to improve the understanding of the high energy Universe. The recent discovery of high energy cosmic neutrinos by IceCube finally established neutrinos as new messengers and began the era of neutrino astronomy.

\section{ANTARES detector}

The ANTARES neutrino telescope in the Northern hemisphere is taking data since more than 10 years and with this monitoring the high energy neutrino sky (see reference [1] for details). The detector is located $40 \mathrm{~km}$ offshore from Toulon at a depth of $2475 \mathrm{~m}$ and comprises 12 mooring lines hosting light sensors (photomultipliers). Its infrastructure also provides opportunities for measurements related to Earth and sea sciences (see e.g. [2]). It also includes an R\&D system of hydrophones to explore the future options of acoustic detection of ultra-high energy neutrinos ([3]).

High energy neutrino interactions are detected via the Cherenkov light caused by the propagation, in water, of relativistic particles created in the interaction. In $v_{\mu}$ charged current interactions muons are created, which can travel for several kilometres through the water and are thus observed as track signatures in the detector. For $v_{e}$ and $v_{\tau}$ charged current interactions and for all flavour neutrino neutral current interactions cascade signatures are detected. Water, as detection medium, allows excellent timing information for the detected light signals, which enables, for all neutrino flavours, a very good angular resolution for the reconstructed direction of detected neutrino candidates. For the direction of muons with energies $>10 \mathrm{TeV}$ the angular resolution reaches 0.4 degrees and for the direction of cascades an angular resolution better than 3 degrees is achieved.

\section{Point Source Searches}

The excellent angular resolution enables for ANTARES powerful searches for neutrino point sources by resolving event excesses at source positions over the approximately isotropically distributed background of atmospheric neutrinos. For the first time ANTARES has performed an all-flavour search for point sources using the data from 2007 to end of 2015 (2424 livedays) [4]. A full sky search was performed, as well as a focussed search at the positions of pre-selected point source candidates (including the IceCube HESE events and HAWC detections), and a dedicated 
search in the Galactic Centre region. For none of the searches a significant excess in events was observed and accordingly upper limits were set on the neutrino fluxes. A skymap with the selected reconstructed origin directions of the 7629 tracks and 180 cascades is displayed on the left side of figure 1 together with the positions of the pre-defined point source candidates. The neutrino flux sensitivity and limits for the point source candidates are shown on the right side of figure 1 in comparison to the recent results by IceCube. The new ANTARES limits are the tightest limits in the Southern Hemisphere with especially good sensitivity at low energies $(<100 \mathrm{TeV})$. From the search in the Galactic Centre region the number of IceCube events originating from a single source in this region can be constrained to at most 3-10, depending on the assumptions on the neutrino energy spectrum.

A common IceCube and ANTARES analysis has been performed using the ANTARES data set from 2008-2012 [5]. The resulting neutrino flux limits in the Southern Hemisphere are around a factor $\sim 1.5$ better than the ANTARES limits alone from the same data period. An update of this analysis with also the recent data from ANTARES and IceCube is ongoing.
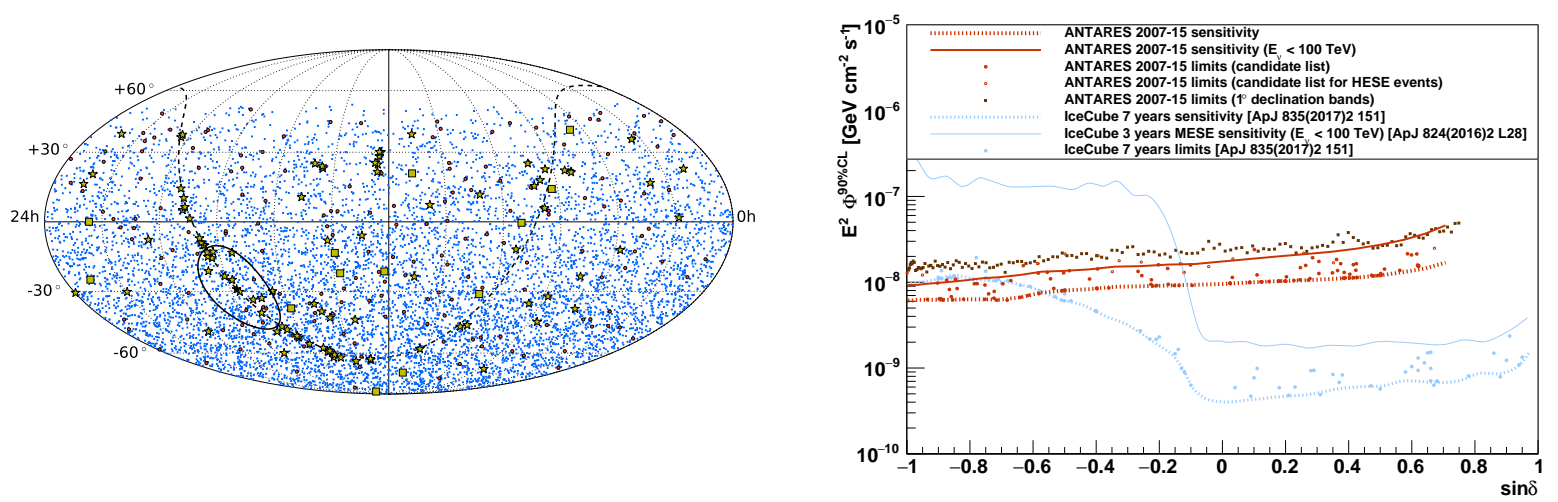

Figure 1: Left: The reconstructed origins of the selected ANTARES neutrino candidates are shown in a skymap in equatorial coordinates. Blue dots represent track events and red dots cascade events. The yellow stars indicate the positions of candidate sources and the yellow squares the positions of the HESE IceCube tracks. The Galactic Centre region is circled by a black line. Right: The ANTARES sensitivity and $90 \%$ confidence upper limits for the point sources are shown as function of $\sin$ (declination) in comparison to the latest IceCube point source results.

\section{Extended Sources}

The ANTARES data were also analyzed searching for potential neutrino emission from known extended structures, namely the so-called Fermi Bubble region and the Galactic Plane emission. The Fermi Bubble is a structure discovered as a region emitting $\gamma$ rays with a hard $\mathrm{E}^{-2}$ spectrum and also observed in microwaves and radio. It consists of two 'bubbles' to the North and South of the Galactic Centre with an overall region of $0.66 \mathrm{sr}$ and its origin is still unclear. Several of the proposed models include hadronic mechanisms with collisions of cosmic-ray protons with interstellar matter, which would also entail neutrino emission. For the analysis of the Fermi Bubble region track events from 2008-2015 were used. The number of events in that region were compared to 
the corresponding number of events in regions, which were equivalent in local coordinates. The selection of events was tuned to maximize the model rejection factor considering only events above a high-energy threshold, where the signal is expected to be most distinct. In the signal region 28 events were observed, while on average 19.7 were expected from the background regions, corresponding to a $1.5 \sigma$ excess. $90 \%$ confidence limits were accordingly set [6]. An update of the analysis using also cascade events is being pursued, whereby it has been estimated that those can add a similar sensitivity.

The Galactic Plane is an interesting target as - aside from the potential neutrino emission from galactic sources - a guaranteed neutrino flux is expected from interactions of cosmic rays with the interstellar medium, however, conventional models predict fluxes below current sensitivities. The new IceCube observations of cosmic neutrinos raised speculations on a potential galactic component in the detected events. The spectral indices of the neutrino energy spectra as observed in the Southern and Northern Hemispheres (in different energy ranges) were found to be different, which could be consistent with a prominent galactic contribution to this flux [8]. The ANTARES data can help probing such scenarios. In a recent all-flavour analysis a new model using a radiallydependent diffusion coefficient has been probed, which is able to account for local cosmic ray as well as Galactic $\gamma$ ray observations [9]. The spatial distribution of the neutrino flux as predicted by this model has been used as a template (left side figure 2) and compared to the observations in a likelihood analysis. No significant excess was found and a limit on the flux was set at $20 \%$ above the model prediction (right side figure 2). This excludes the diffuse Galactic neutrino emission as the major cause of the "spectral anomaly" between the two hemispheres measured by IceCube and restricts the galactic contribution in the HESE sample at 90\% CL to less than 10\%. Also this analysis is being followed up in a combined analysis with IceCube.
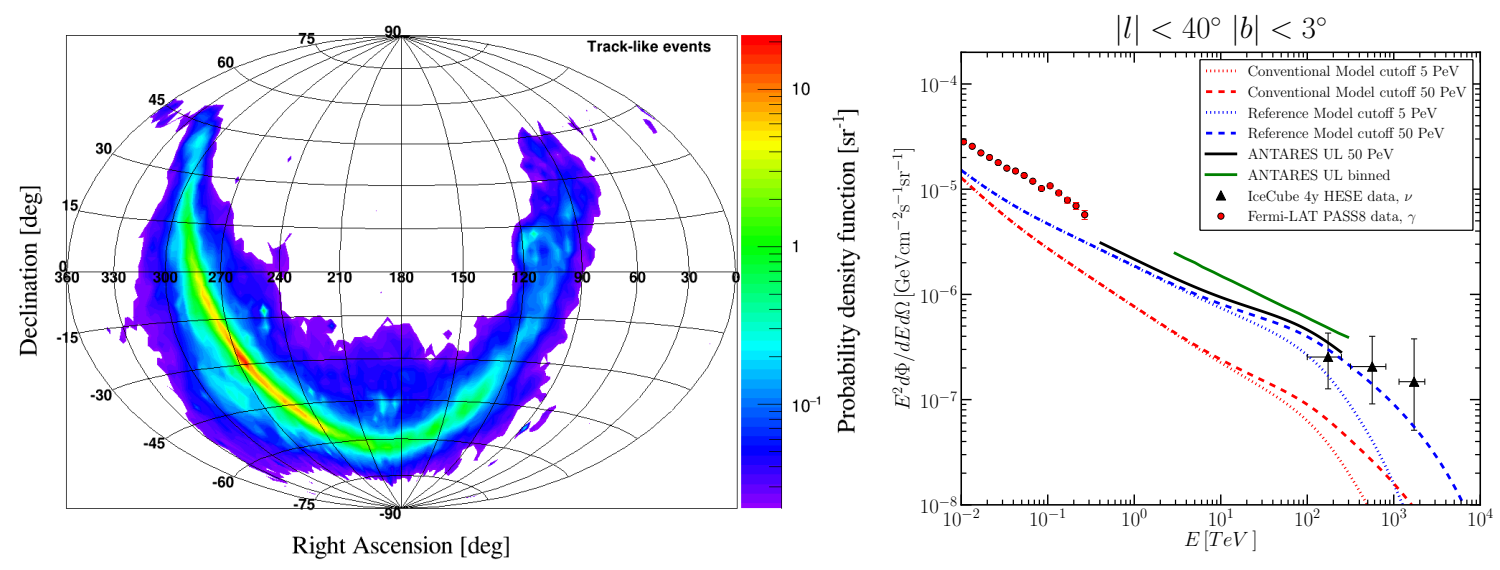

Figure 2: Left: The normalized probability distribution of track directions in the ANTARES detector as predicted by the probed model of a diffuse emission in the galactic plane. Right: ANTARES upper limit at $90 \%$ confidence level on the three flavour neutrino flux (solid black line) on the reference model with a 50 PeV energy cut-off ([10]).

\section{Diffuse Flux}

The search in ANTARES for a diffuse flux of cosmic neutrinos has been pursued using all 
flavour neutrino candidates with an optimized selection for events with high energies, where the flux is best distinguishable from the atmospheric background. In the final selection $13.5 \pm 3(5 \pm 2)$ track (cascade) events were expected from background and 3(1.5) events from the diffuse flux of cosmic neutrinos, as observed by IceCube, while 19 (7) events were observed in the data for tracks (cascades), consistent with both background only and background plus a cosmic contribution. The resulting upper limits are shown in figure 3 [7]. The analysis is continued to include also the latest data from the cascades.

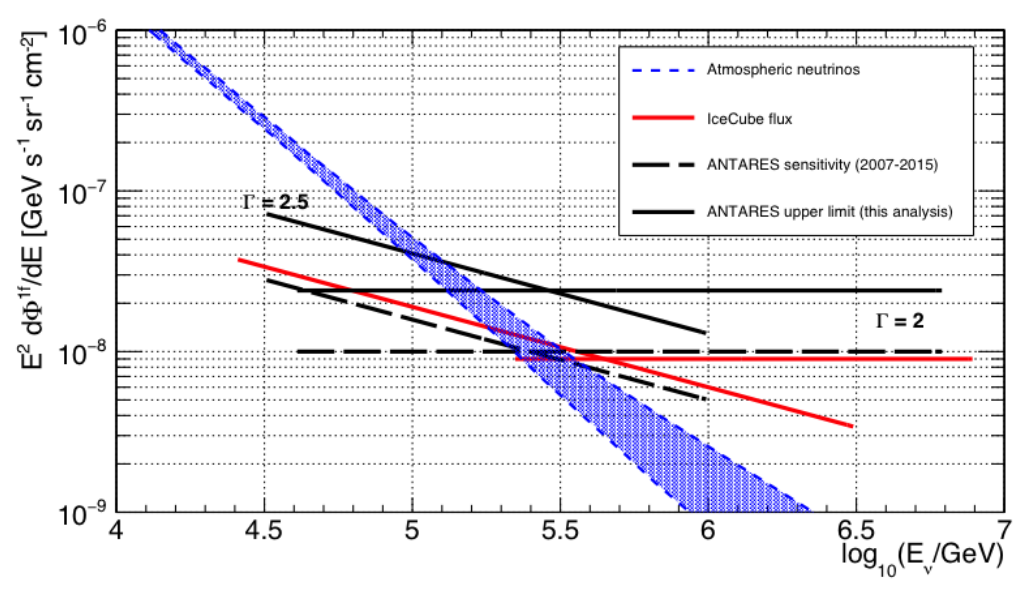

Figure 3: ANTARES sensitivity and $90 \%$ confidence upper limits on the diffuse neutrino flux are shown for both an $E^{-2}$ and $E^{-2.5}$ spectrum in comparison to the measured IceCube flux.

\section{Multimessenger observations}

ANTARES is embedded in a network of other observatories ranging from electromagnetic observatories (optical, radio, X-ray, $\gamma$ rays) to cosmic ray and gravitational wave detectors sharing alerts. More than 200 alerts have been sent within TaToO (Telescopes-ANTARES Target of Opportunity [11]) to optical robotic telescopes (TAROT, ROTSE and MASTER) since 2009 and 12 for X-ray observations with the XRT instrument on board the Swift satellite since 2013. Also the Murchinson Widefield Array (MWA, radio) followed up on two ANTARES alerts, H.E.S.S. followed up on two alerts and HAWC on 36 since 2014 [12].

Dedicated analyses in ANTARES follow-up on alerts distributed through the Gamma-ray Coordinated Network (GCN) to look for transient events. When Gamma Ray Burst (GRB) alerts are received by ANTARES special data taking is invoked, enabling the storage of up to two minutes of raw unfiltered data. Recently these raw data files have been analysed with a dedicated reconstruction to assess the potential neutrino signal from four of the brightest Gamma Ray Bursts and especially probe models, which expect also enhanced low energy neutrino emission. No signals were found and thus corresponding limits were set [13]. 
Also the high energy neutrino alerts by IceCube and the alerts from LIGO and Virgo on gravitational wave candidates are being followed up in real-time and in refined offline analyses. In a common effort the data from ANTARES and IceCube have been searched for neutrinos accompanying the prominent first gravitational wave event GW150914 [14]. No neutrinos were found in the ANTARES event selection within \pm 500 seconds around the event (consistent with the background assumption) and combined with the result from IceCube (3 events consistent with background) limits were set on the maximal neutrino flux in that time window. The $90 \%$ confidence limits are shown in figure 4 for an $E^{-2}$ flux as well as for an $E^{-2}$ flux with a $100 \mathrm{TeV}$ energy cutoff. The ANTARES limits dominate in the Southern Hemisphere for the softer spectrum with the cutoff. Analyses are underway to probe the neutrino flux associated with further gravitational wave candidates. are already
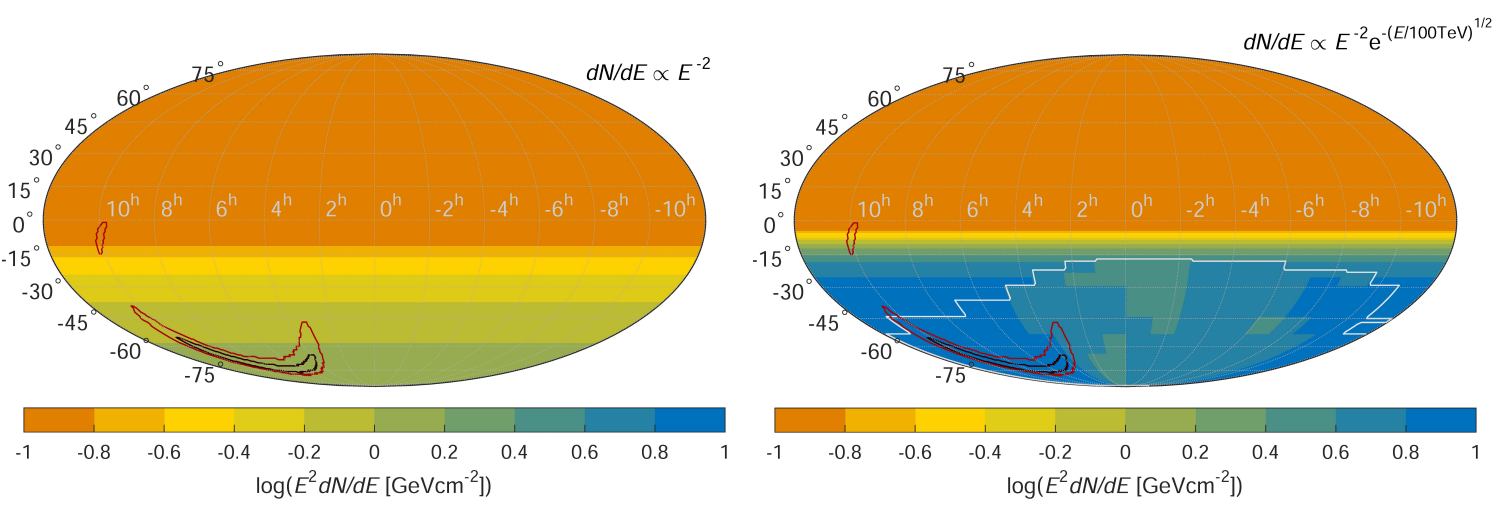

Figure 4: Upper limits on the neutrino fluence for the gravitational wave candidate GW150914 as function of direction in equatorial coordinates for an $E^{-2}$ spectrum (with $100 \mathrm{TeV}$ cutoff on the right side). The region surrounded by a whte line shows the part of the sky in which ANTARES is more sensitive. For comparison, the 50\% and $90 \%$ CL contours of the gravitational wave sky map are also shown.

\section{Dark Matter and exotics}

In WIMP (Weak Interacting Massive Particles) dark-matter scenarios it is expected that WIMPs cluster in massive objects like the Sun, Earth, Galactic Centre and Dwarf galaxies, where then annihilation takes place. The annihilation products can be neutrinos, but also other particles, which subsequently produce neutrinos in their decays, which allows for neutrino telescopes to probe some phase space of WIMP dark matter models. The search for a potential neutrino flux from WIMP annihilation in the Sun achieved a sensitivity on the spin-dependent WIMP interaction cross section, $\sigma_{S D}$, which complements the results from direct dark matter searches. The recent ANTARES limits can be seen together with the results from other experiments in the left side of figure 5 .

ANTARES, thanks to its direct view of the Galactic Centre and to its excellent angular resolution, could also place for high WIMP masses the most stringent limits from neutrino observations on the WIMP-WIMP velocity-averaged self-annihilation cross section $\langle\sigma v\rangle$ from the Galactic Centre [16]. In addition WIMP signatures from the centre of the Earth and secluded dark-matter models in the Sun have been probed and limits been set accordingly [17]. 
ANTARES is also pursuing a new neutrino oscillation analysis with sensitivity on sterile neutrinos and has placed limits in searches for magnetic monopoles and nuclearites [18].
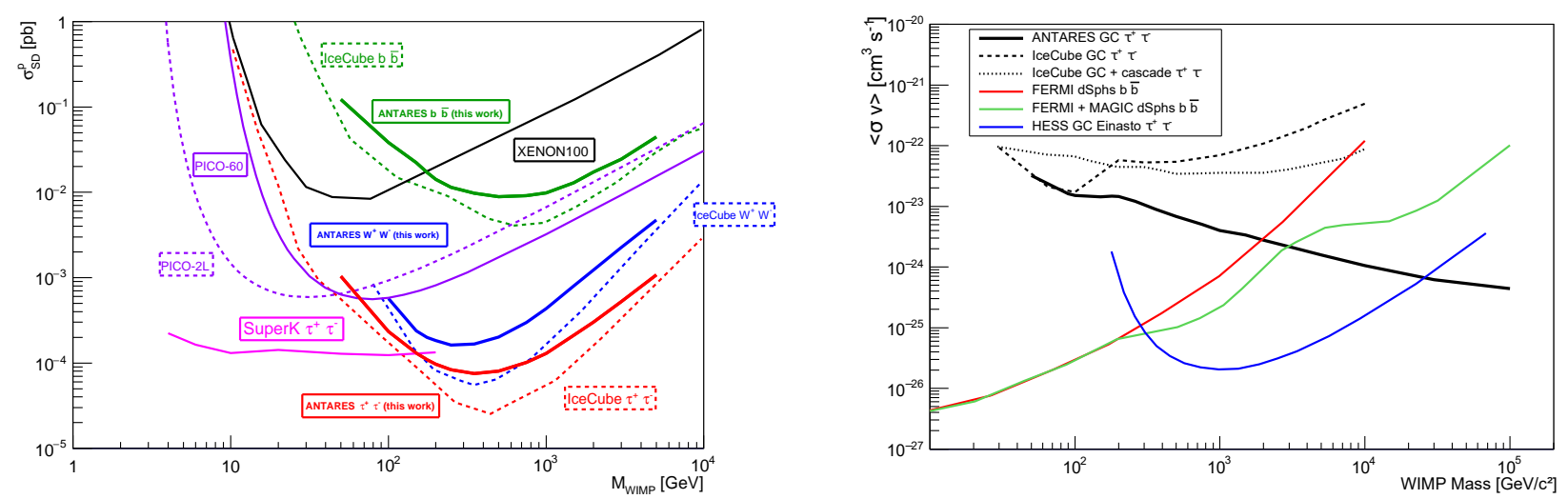

Figure 5: Left: ANTARES limits from the Sun on the spin dependent WIMP interaction cross section are shown as function on the WIMP mass as red, green and blue solid lines in comparison to the results of other experiments [15]. Right: ANTARES limits from Galactic Centre observations on the velocity averaged WIMP interaction cross section are shown for the decay to $\tau^{+} \tau^{-}$as function of the WIMP mass in comparison to the results of other experiments [16].

\section{Conclusion and outlook}

The ANTARES neutrino telescope has been running successfully for over 10 years with a variety of interesting physics results. Especially its excellent angular resolution for all flavour neutrino interactions enables sensitive point source searches and its direct view of the Galactic Centre region access to an interesting sky region. Analyses are also underway in combination with IceCube to optimally exploit all information available from the high energy neutrino sky. Construction has by now started for the KM3NeT neutrino telescope in the Mediterranean Sea, which is building on this heritage to explore in the future the high energy neutrino sky with enhanced sensitivity and resolution, promising an exciting future for neutrino astronomy. 


\section{References}

[1] M. Ageron et al., Nucl. Inst. Meth. A 656 (2011) 11

[2] C. Tamburini, et al., PLoS ONE 8(7): e67523. doi:10.1371/journal.pone.0067523 (2013)

[3] J. A. Aguilar et al., Nuclear Inst. and Meth.A 626 (2011) 12

[4] A. Albert et al., arXiv:1706.01857 (2017)

[5] S. Adrián-Martínez, et al, The Astrophys. J. 823 (2016) 65

[6] S. Hallmann et al (2017) J. Phys.: Conf. Ser. 888 012102, poster NEUTRINO 2016

[7] L. Fusco et al., EPJ Web of Conferences 136, 04005 (2017), RICAP2016

[8] M.G. Aartsen et al., Astrophys.J. 833 (2016) no.1, 3

[9] D. Gaggero et al., Astrophys. J. Lett., 815:L25 (2015)

[10] A. Albert et al., Phys. Rev. D. 96 (2017) 062001

[11] M. Ageron et al., Astropart. Phys. 35 (2012), 530-536

[12] A. Coleiro, EPJ Web of Conferences 136, 04003 (2017)

[13] A. Albert et al., MNRAS 469 (2017) 906-915

[14] S. Adrián-Martínez et al., Phys. Rev. D 93 (2016) 122010

[15] S. Adrián-Martínez et al., Phys. Rev. Lett. B 759 (2016) 69

[16] A. Albert et al., Phys. Lett. B 769 (2017), 249

[17] A. Albert et al., Physics of the Dark Universe 16 (2017) 41-48

[18] A. Albert et al., J. High Energ. Phys. (2017) 2017:54, G. Pavalas PoS (ICRC2015) 1060 (2015) 